\title{
Study on the Effect of Removing the Slide Wire Screw on Locking Plate by Nail Groove Reconstruction Method
}

\section{Guanglei Zheng}

Hangzhou No 2 Peoplel's Hospital: Hangzhou Normal University Affiliated Hospital https://orcid.org/0000-0002-7824-5353

\section{Yingjie Qi ( $\nabla$ yjqiosteo@126.com )}

Hangzhou No 2 Peoplel's Hospital: Hangzhou Normal University Affiliated Hospital https://orcid.org/0000-0002-9532-9233

\section{Research Article}

Keywords: fracture fixation, internal, Locking screws, Sliding wire, difficult removal

Posted Date: December 13th, 2021

DOI: https://doi.org/10.21203/rs.3.rs-1121716/v1

License: (a) (i) This work is licensed under a Creative Commons Attribution 4.0 International License. Read Full License 


\section{Title:}

2 Study on the effect of removing the slide wire screw on locking plate by nail groove

3 reconstruction method

\section{Authors:}

5 Guanglei Zheng ${ }^{1}$, Yingjie $\mathrm{Qi}^{1, *}$

$6{ }^{1}$ Department of Orthopaedics, The Affiliated Hospital of Hangzhou Normal

7 University, Hangzhou 310000, China

$8{ }^{*}$ Correspondence to: Professor Yingjie Qi. The Affiliated Hospital of Hangzhou

9 Normal University, 126 Wenzhou Road, Hangzhou 310000, China. E-mail: yjqiosteo@126.com

11 Abstract

Background: This paper is to describe and evaluate the nail groove reconstruction method in removing slide wire screw on locking plate. Then compare the method with tungsten steel drilling nail method, to explore a new method of removing slide wire screw on locking plate.

Method: A total of 1254 patients with removal fracture internal fixation devices were collected from the Affiliated Hospital of Hangzhou Normal University from July 2015 to September 2021, of which 62 cases met the inclusion and exclusion criteria. All patients were randomly divided into the experimental group and the control group. 31 people per group. There were 19 males and 12 females in the experimental group, the age of patients was $35.68 \pm 11.70$ years; while 18 males and 13 females in the control group, the age of patients was $36.27 \pm 10.37$ years. Nail groove reconstruction 
1 method was used in the experimental group, and the tungsten steel drilling nail

2 method was used in the control group. Collect and count surgical-related indicators,

3 the data of two groups were compared and analyzed from four aspects: intraoperative

4 blood loss, operation time, incision healing time and limb function recovery time.

5 Result: All slide wire screws were removed successfully, and all patients had no

6 serious postoperative complications such as internal fixation retention and

7 neurovascular injury. The experimental group was better than the control group in the

8 following three aspects: the amount of intraoperative blood loss, the operative time,

9 the recovery time of limb function, and the differences were statistically significant $(\mathrm{p}$

$10<0.05)$. There was no significant difference in incision healing time between the two

11 groups.

12 Conclusion: The nail groove reconstruction method has less damage to the bone 13 and soft tissue, less intraoperative blood loss, shorter operation time, and faster postoperative recovery of limb function. The nail groove reconstruction method is a 15 simple and effective method, it has obvious advantages compared with the traditional method.

Keywords: fracture fixation, internal; Locking screws; Sliding wire; difficult removal 


\section{1. Background:}

2 In recent years, open reduction and internal fixation of fracture has been widely used

3 in orthopedics, and the application of locking plate and screw makes most of the

4 fractures get satisfied reduction and fixation. The appropriate use of internal fixation

5 system not only provides strong support for the fracture, but also disperses the stress,

6 which provides a good biomechanical environment for postoperative functional

7 exercise. So that the limb's function can be better restored. Most of the internal

8 fixation need to be removed after fracture healing, especially those related to

9 intra-articular fractures. The studies show that the pain is relieved and the function is

10 significantly improved after removing internal fixation of ankle joint and knee joint

11 (1-3). Although the removal of internal fixation device is a simple operation,

12 according to statistics, the incidence of complications is as high as $20 \%$, among which

13 the difficulty caused by slide wire screws is the most common and most troublesome

14 problem for clinicians (4-6). When screw slipping occurs during operation, improper

15 treatment may cause a series of iatrogenic injuries, including postoperative fracture,

16 neurovascular injury, and increase the probability of postoperative infection (7). At

17 present, there are the following common methods are used to remove slide wire

18 screws: (1)Rotating plate loosening screw method, (2)Bending or shearing steel plate

19 method, (3)Cutting off the end of screws method, (4)Groove-changing method.

20 However, these methods have some limitations and shortcomings, such as they can't

21 be used in the case of two or more adjacent screws sliding wire, and a lot of metal

22 debris will be produced in the process of screw extraction. In response to the dilemma 
1 in the clinic, inspired by the groove-changing method, we designed a new method,

2 called nail groove reconstruction method.

3 We aim the large-sized screwdriver at the groove of the slide wire screw and slowly

4 knock it in along the direction of the screw to make a new torx groove in the tail of

5 the screw. Keep the screwdriver always in the direction of the screw during the

6 tapping process. When the screwdriver is completely in the groove, turn the

7 screwdriver to remove the slide wire screw. This method can take out multiple slide

8 wire screws on any position of the plate. In this study, the nail groove reconstruction

9 method was compared with the traditional method, and its clinical effect was

10 evaluated from the aspects of incision healing time, operation time, blood loss,

11 postoperative limb function recovery and postoperative complications. The purpose of

12 this paper is to explore the advantages and disadvantages of the nail groove

13 reconstruction method and to provide a more safe and effective method for removing

14 slid wire screws.

15 2. Materials and methods

$16 \quad 2.1$ Ethics statement

17 This study was approved by the Ethics Committee of The Affiliated Hospital of

18 Hangzhou Normal University, China. Written informed consent was obtained from all 19 patients.

\subsection{Subjects}

From July 2015 to September 2021, a total of 1254 Patients who underwent the 
1 Hangzhou Normal University were enrolled in this study. 62 patients who met the

2 inclusion criteria were selected. A total of 78 slide wire screws were included. These

3 patients were randomly divided into the experimental group (the nail groove

4 reconstruction method) and the control group (the tungsten steel drilling nail method).

5 There were 19 males and 12 females in the experimental group, the age of patients

6 was $35.68 \pm 11.70$ years, the time after internal fixation was $13.90 \pm 1.89$.

7 Experimental group contained 38 screws, including 4 screws with $5 \mathrm{~mm}$ diameter and

834 screws with $3.5 \mathrm{~mm}$ diameter. Besides, There were 18 males and 13 females in the

9 control group, the age of patients was $36.27 \pm 10.37$, the time after internal fixation

was $14.10 \pm 2.12$. Control group contained 40 screws, including 5 screws with $5 \mathrm{~mm}$

11 diameter and 35 screws with $3.5 \mathrm{~mm}$ diameter. All slide wire screws were hexagonal

12 screws. There was no significant difference between the two groups on the patients'

13 basic information. (including gender, age, time after internal fixation, type and

14 number of slide wire screws $)(p>0.05$, Table 1$)$.

$15 \quad 2.3$ Surgical methods

\subsubsection{Preoperative preparation}

17 Complete the necessarily preoperative examination and preparation: the X-ray or CT

18 of fracture position within one month, Screwdriver corresponding to screw. A

corresponding Torx screwdriver and another larger Torx screwdriver are needed in the experimental group. In this study, operations are performed by the same group of doctors.

\subsubsection{Anesthesia and posture}


1 Patients with fracture of humerus, ulna, radius and clavicle were in the supine position

2 after nerve block anesthesia or general anesthesia. Patients with fractures of femur、

3 tibia or fibula were in the supine position or lateral position after epidural anesthesia

4 or general anesthesia.

\section{$5 \quad$ 2.3.3 Surgical operation}

6 We cut the skin along the original incision, separated the soft tissue to fully expose the

7 plate and screw, and removed the soft tissue and hyperplastic callus embedded around

8 plate and screws. We needed select a matching screwdriver, fully inserted it into the

9 groove of the screw, and removed the screws one by one. If there was screw difficult to remove, put it on hold and removed the easy screw first. Then we tried to remove

11 the difficult screw with T-handle's screwdriver again. When the screw slid, different 12 screw extraction methods were adopted according to the different groups.

Traditional surgery: The tungsten steel drilling nail method was used in the control group. First, we covered the soft tissue around the screw with wet gauze. Then, removed the plate after knocking off the tail of screw by the tungsten steel drill and enlarged the nail path with the ring drill. Finally, the slide wire screw was removed with a nail extractor or vice. In order to prevent metal debris from being left in the body, a large amount of normal saline was needed to rinse the operation area.

Nail groove reconstruction method: The nail groove reconstruction method was used in the experimental group. The larger toxr screwdriver was knocked into the groove of the slide wire screw. When the larger toxr screwdriver was absolutely advanced into the screw groove, a new torx groove was reconstructed. Then we could 
1 turn the screwdriver to remove the screw slowly. There were a few points for attention:

2 (1)Keep the screwdriver in the same direction as the screw; (2)Be gentle and slow in

3 the process of knocking; (3)Pay attention to the metal debris in the process. The

4 screwdriver used during the operation is shown in Figure 1, and the typical case is

5 shown in Figure 2-5.

$6 \quad$ 2.3.4 Postoperative treatment and rehabilitation management

7 Patients in both groups were given non-steroidal anti-inflammatory drugs (NSAIDs)

8 after surgery for postoperative early activity. The drainage device was removed and

9 X-ray or CT reexamination was performed within 24 hours postoperatively. In the case of ensuring no re-fractures, We began to instruct patients to do functional exercise. Outpatient follow-up should be carried out every two weeks.

\subsection{Outcome measures:}

In this study, the results of the experimental group and the control group were compared and analyzed from four aspects: intraoperative blood loss, operation time, incision healing time and limb function recovery time.

The quantity of intraoperative blood loss referred to the amount of blood loss removing a slide wire screw. Operation time referred to the time it took to remove a slide wire screw. Incision healing time referred to the number of days from the first postoperative day to the healing of incision grade $\mathrm{A}$. The limb function recovery time referred to the number of days from the first postoperative day to the preoperative level.

Postoperative follow-up was mainly based on two methods: telephone follow-up and 
1 outpatient reexamination. All patients were instructed to return to the outpatient clinic

2 every two weeks after operation. We determined whether the patient had

3 postoperative complications such as re-fracture, osteomyelitis, joint stiffness,

4 limitation of joint movement through imaging examination and physical examination.

\section{$5 \quad 2.5$ Statistical analysis :}

6 The data were processed and analyzed by SPSS 23.0 statistical analysis software

7 (IBM Corporation, New York, NY). The measurement data were presented as $\bar{X} \pm \mathrm{S}$.

8 The difference between two groups was compared by the independent sample t-test.

9 The Chi-square test was performed for the qualitative data. Statistical significance

10 was set at $P$ value less than 0.05 .

\section{Results}

A total of 62 patients were included in this study, 31 patients per group. The intraoperative blood loss in the experimental group $(116.13 \pm 21.65)$ was less than that in the control group $(168.06 \pm 29.67)$. The difference is statistically significant ( $p$ $<0.05)$. The operation time of the experimental group was $28.94 \pm 4.61$, and this in the control group was $42.10 \pm 5.74$. Obviously, the operation time in experimental group was significantly shorter than that in the control group $(\mathrm{p}<0.05)$. The incision healing time of the experimental group and the controlled group were $13.74 \pm 1.44$ and $13.61 \pm 1.26$, respectively, with no significant difference between them $(p>0.05)$. The recovery time of limb function in the experimental group was $19.52 \pm 3.04$, the control group was $29.16 \pm 4.39$. There was significant difference between the two groups $(\mathrm{p}<0.05)$ (Details in Table 2 and Figure 6). 
1 All internal fixations were removed in all cases. The incisions of all patients were

2 healed by I / A, and there were no incision complications such as infection, effusion,

3 hematoma and so on. It was found that all patients' limb function could be restored to

4 the preoperative level and there were no complications such as neurovascular injury

5 in the postoperative follow-up.

\section{4. Discussion}

7 In recent years, with the application of locking plate in orthopedics, the bone healing

8 rate and postoperative limb function have been significantly improved. In China, most

9 of patients ask doctor to remove internal fixation devices after fracture healing. The removal of internal fixation device is the low-level operation, but when the screw is

11 slippery, the difficulty of the operation is greatly increased. When it is difficult to remove plates and screws, improper removal will cause complications such as re-fracture, neurovascular injury, retention of internal fixation and so on( 7). There are so many reasons for screw slipping, which can be summarized three aspects: Patient factors, Internal fixation factors and Latrogenic factors. 1. Patient factors. Maehara et al. showed that the bone of young patients was hard and firmly combined with screws, so it was difficult to remove, and the screw in cadre was more difficult to remove than the metaphysis (8). A number of studies have shown that the longer the screw is fixed, the greater the stress and the more prone to deformation. And the screw is buried by hyperplastic bone, which greatly increases the resistance when the screw is removed, and eventually leads to screw slipping $\quad(5,8)$.2. Internal fixation factors. Screws in the lower extremities are generally longer than those in the upper 
1 extremities. And the thicker and denser cortex of the lower extremity bone makes it

2 have greater biomechanical strength in conjunction with the screws, so slide wire is

3 easy to occur when removed $(9,10)$. Several studies have shown that the $3.5 \mathrm{~mm}$

4 screw is more difficult to be removed than $4.5 \mathrm{~mm}$ and $5.0 \mathrm{~mm}$ screw (5). Statistics

5 show that the rate of $3.5 \mathrm{~mm}$ screw slide wire is $10.7 \%$, while that of $5.0 \mathrm{~mm}$ screw is

$62.0 \%$. The difference is statistically significant. The main reason is that the groove of

$7 \quad 3.5 \mathrm{~mm}$ screw is shallow and small (8). Hayes et al. have shown that the screw with

8 surface polishing is easier to remove (7). The application of locking plate

9 significantly increases the incidence of slide wire screw $(5,6)$. The locking plate 10 commonly is made by titanium alloy and stainless steel. And the biological 11 characteristic of titanium alloy is more consistent with the bone, but the hardness is 12 low, which can easily lead to screw deformation and wear when encountered with 13 resistance $(4,5,8,11)$. And the cold welding effect will occur between the titanium 14 alloy plate and screw, so titanium alloy screw is most likely to slide wire $(12,13)$. 3 . 15 Latrogenic factors. The irregular operation of the surgeon is an important reason 16 leading to slide wire screw, including: (1)The drill bit didn't match the screw; (2)The 17 sight was not used when drilling the screw was not placed in the direction of the 18 screw path, and no torque limiting screwdriver was used; (3)The screwdriver was not 19 in the same direction as the screw, resulting in wear at the tail of the screw; (4)The 20 soft tissue or hyperplastic bone in the groove was not cleaned when the nail was 21 removed $(13,14)$; (5)When the combination of the screw and the plate is over 22 tightened, the surgeon tried to use a power tool to remove the screw instead of the 
T-handle screwdriver (15).

In recent years, with the increasing preference of clinicians for locking plate and screw system, the incidence of slide wire screw has increased significantly, which has also attracted the attention of orthopedic surgeons. Through continuous research, improvement and practice, many scholars have put forward a variety of methods to remove slide wire screws. We classify the existing mainstream methods into the following categories: 1. Cutting off the tail and taking screw. After destroying the tail of the screw with a tungsten steel drill, the plate is removed first, and then the screw is removed by expanding the screw path with a ring drill or Kirschner needle. Gupta improved this method by directly grinding the plate along the screw direction with a grinding drill, and then removing the plate and screws. This method can remove multiple screws without being restricted by the position of the screws. But it takes a lot of time, and a large amount of metal debris will be produced in the process, which is often stranded in the patient's body (10). And the method will damage the normal bone and reduce the bone strength, which is not conducive to early postoperatively functional exercise. 2. Integration of the plate and screws. There are many kinds of methods, all of which are to pry and loosen the steel plate and directly rotate the plate to remove them together. This method does not damage normal bone, but it is generally limited to single slide wire screw. And in the course of operation, it may be necessary to extend the incision and increase the damage to the surrounding soft tissue. Although Gopinathan et al. (9) solved the problem of several screws slipping by cutting the plate on both sides of the slide wire screw, it might take a lot of 
1 time to cut off the plate in the femur or tibia. Therefore this method is not suitable for

2 femur and tibia. In addition, Kim et al. (16) invented a longitudinal drilling method,

3 which was only for the case of screw slipping in the locking- compression hole. 3.

4 Groove-changing method. Make a new "one-shaped" groove at the tail of the slide

5 wire screw and remove the screw with a corresponding screwdriver. This method will

6 produce a large amount of metal debris in the process, and the new groove is

7 generally shallow and irregular, so that the friction force produced by the screwdriver

8 is low. The above methods all have some limitations and shortcomings. By

9 summarizing up the experiences and reviewing the relevant literature, we put forward the nail groove reconstruction method on the basis of the Groove-changing method.

11 Through the control study, it is found that it has a good effect.

12 From the results, we can see that all slide wire screws can be removed in both of the experimental group and the control group, and there is little difference in the success rate of operation. However, in terms of operation time, intraoperative blood loss and limb function recovery time, the experimental group is better than the control group, and the difference is statistically significant $(\mathrm{P}<0.05)$. Compared with the patients who accept the traditional method, the patients with the nail groove reconstruction method has shorter operation time, less intraoperative blood loss and lighter postoperative pain, which is beneficial for them to do postoperatively functional exercise in the early stage. Especially for the patients with lower limb fracture, the traditional method often has obviously postoperative pain, while clinicians worry about the weakening of bone strength and ask patients to delay the time of limb 
1 weight-bearing. In another word, the patients are unable to accomplish the early

2 weight-bearing exercise. By analyzing the above results, we verify the clinical

3 feasibility of the nail groove reconstruction method, and summarize the following

4 three advantages. 1. Simple and easy. No special tool is needed, and the method

5 is easy to master. 2. The operation time is short, the bleeding is less, and the risk of

6 infection is reduced. 3. It does not cause damage to the normal bone, does not reduce

7 the bone's strength, and the patients feel less pain after the operation, which is

8 conducive to early postoperative exercise to obtain better function. At the same time,

9 we would like to remind you to pay attention to the following three points when using

10 the nail groove reconstruction method. 1. For patients with osteoporosis, the new

11 groove needs to be rebuilt gently and slowly in order to avoid fracture. 2. When the

12 screwdriver is knocked into the groove, you need to keep it in the same direction as

13 the screw in order to get the maximum friction. 3. Spread gauze around the screw to

14 prevent the metal detritus from being left in the body.

15 Slide wire screw is usually caused by one or more reasons. It is difficult to predict the

16 occurrence of slide wire screw before operation, but we can take some measures to

17 reduce the probability. For example, avoid implanting the screw with power tools, use

18 the torque-limited screwdriver, and keep the direction of the screw is consistent with

19 the path. X-ray must be performed before the operation, and CT should be done if

20 possible, so that we can better see the situation of callus embedding around the plate

21 and screws. The bone callus and soft tissue in the groove should be removed, the

22 screwdriver should be full contact with the screw and in the same direction of the 
1 screw. It is best to use the T-handle screwdriver to get as much friction as possible.

2 Although we have made sufficient preoperative preparations and standard operation,

3 it is inevitable that the screw will slide wire. When it is confirmed that the screw

4 cannot be removed, you should communicate with the patient and his family and give

5 up removing the screw in order to avoid serious complications.

6 5. Conclusion:

7 This control study shows that the nail groove reconstruction method can be used to

8 remove the slide wire screw that is on the locking plate, especially for hexagonal

9 screws with $3.5 \mathrm{~mm}$ diameter. Furthermore, compared with the traditional methods, the nail groove reconstruction method has the advantages of small trauma, simple operation, quick recovery, safe and effective.

\section{List of abbreviations:}

Open reduction and internal fixation(ORIF) 


\section{Declarations}

2 Ethics approval and consent to participate:

3 This study was conducted at the Affiliated Hospital of Hangzhou Normal University,

4 and permission was obtained from the hospital's Ethics Committee. The authors had

5 obtained patients' consents before enrolling the participants.

6 Consent for publication:

$7 \quad$ Not applicable

8 Availability of data and materials:

9 The data and materials might be obtained from the corresponding author upon 10 request.

11 Competing interests:

12 The authors declare that they have no competing interests.

13 Funding:

14 Not applicable.

15 Authors' contributions:

YJ.Qi contributed to the conception and design of the study. The study was performed by GL.Zheng and YJ.Qi. And GL.Zheng also contributed to the data acquisition and analysis, and manuscript writing. Both authors read and approved the final manuscript.

\section{Acknowledgements:}

I would like to thank my lover YQ.Li for her support to my work and Director CC.Liu for his guidance on my surgical techniques. 


\section{References}

2 1. Garner MR, Thacher RR, Ni A, et al. Elective removal of implants after open reduction and

3 internal fixation of Tibial Plateau fractures improves clinical outcomes. Arch Orthop Trauma

$4 \quad$ Surg.2015;135:1491-6.

5 2. Reith G, Schmitz-Greven V, Hensel KO, et al. Metal implant removal: benefits and 6 drawbacks--a patient survey. BMC Surg.2015;15:96.

7 3. Williams AA, Witten DM, Duester R, et al. The benefits of implant removal from the foot 8 and ankle. J Bone Joint Surg Am.2012;94:1316-20.

9 4. Georgiadis G, Gove N, Smith A, et al. Removal of the less invasive stabilization system. Journal of orthopaedic trauma.2004;18:562-4.

11 5. Bae JH, Oh JK, Oh CW, et al. Technical difficulties of removal of locking screw after locking 12 compression plating. Arch Orthop Trauma Surg.2009;129:91-5.

6. Tan SL, Balogh ZJ. Indications and limitations of locked plating. Injury.2009;40:683-91.

7. Raja S, Imbuldeniya AM, Garg S, et al. Difficulties encountered removing locked plates. Ann R Coll Surg Engl.2012;94:502-5.

8. Maehara T, Moritani S, Ikuma H, et al. Difficulties in removal of the titanium locking plate in Japan. Injury.2013;44:1122-6.

9. Gopinathan N, Dhillon M, Kumar R. surgical technique: simple technique for removing a locking reconplate with damaged screw heads. Clinical orthopaedics and related research.2013;471:1572-5. easy, quick, and safe technique. J Surg Tech Case Rep.2014;6:9-11. 
1 11. Christensen FB, Dalstra M, Sejling F, et al. Titanium-alloy enhances bone-pedicle screw

2 fixation: mechanical and histomorphometrical results of titanium-alloy versus stainless steel. Eur

3 Spine J.2000;9:97-103.

4 12. Schwarz N, Euler S, Schlittler M, et al. Technical complications during removal of locking 5 screws from locking compression plates: a prospective multicenter study. European Journal of

6 Trauma and Emergency Surgery.2013;39:339-44.

7 13. Musters GD, Boele van Hensbroek P, Ponsen KJ, et al. Locking Compression Plates are more

8 difficult to remove than conventional non-locking plates. European Journal of Trauma and $9 \quad$ Emergency Surgery.2013;39:159-62.

14. Hontzsch D, Stuby FM. Removal of plates and screws. Tips and tricks for problematic cases.

Unfallchirurg.2012;115:291-8.

15. Nizaj N, Shamseer CM, Sukesh AN, et al. Manual extraction is superior to power tools when removing stripped titanium locking screws. Injury.2021.

16. Kim SJ, Kim MU. A simple technique for removing a locking compression plate with a stripped locking screw. J Orthop Trauma.2012;26:e51-3.

(1)

(1)

(1)




\section{Tables:}

2

3

4

5 Table 1. Comparison of patients' basic information between both groups

\begin{tabular}{ccc}
\hline & Experimental group & Control group \\
\hline Case (n) & 31 & 31 \\
Male (n) & 19 & 18 \\
Female (n) & 12 & 13 \\
Age (year) & $35.68 \pm 11.70$ & $36.27 \pm 10.37$ \\
Time after ORIF (m) & $13.90 \pm 1.89$ & $14.10 \pm 2.12$ \\
$3.5 \mathrm{~mm}$ diameter screws (n) & 34 & 35 \\
$5.0 \mathrm{~mm}$ diameter screws (n) & 4 & 5 \\
\hline
\end{tabular}

6

Note: All slide wire screws were hexagonal screws

7

8

9

10

11

12

13 
Table 2. Comparison of surgical indicators between both groups

\begin{tabular}{ccc}
\hline & Experimental group & Control group \\
blood loss (ml) & $116.13 \pm 21.65^{*}$ & $168.06 \pm 29.67$ \\
operation time (min) & $28.94 \pm 4.61^{*}$ & $42.10 \pm 5.74$. \\
incision healing time (d) & $13.74 \pm 1.44$ & $13.61 \pm 1.26$ \\
function recovery time (d) & $19.52 \pm 3.04^{*}$ & $29.16 \pm 4.39$ \\
\hline
\end{tabular}

7 Note: $*$ Compared with the control group, $\mathrm{P}<0.05$. The incisions of all patients got

8 grade $\mathrm{A}$ healing, and there were no complications

9

10

11

12

13

14 
1 Figure Legends:

2

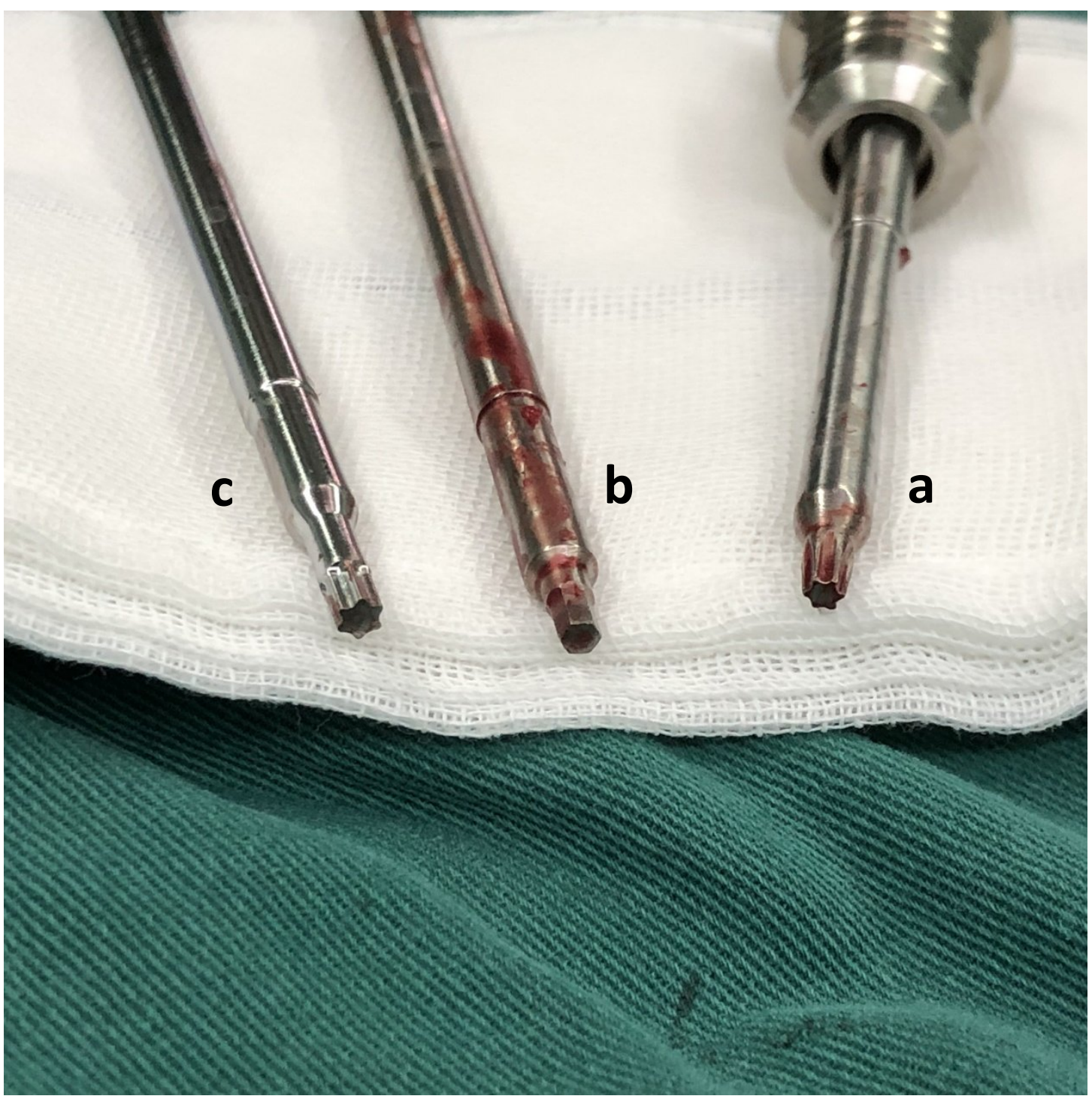

4

Fig.1: The screwdrivers used during the operation.

$5 \mathrm{a}$ and $\mathrm{b}$ is for screws with $3.5 \mathrm{~mm}$ diameter, $\mathrm{c}$ is the larger toxr screwdriver

6

7

8

9

10 


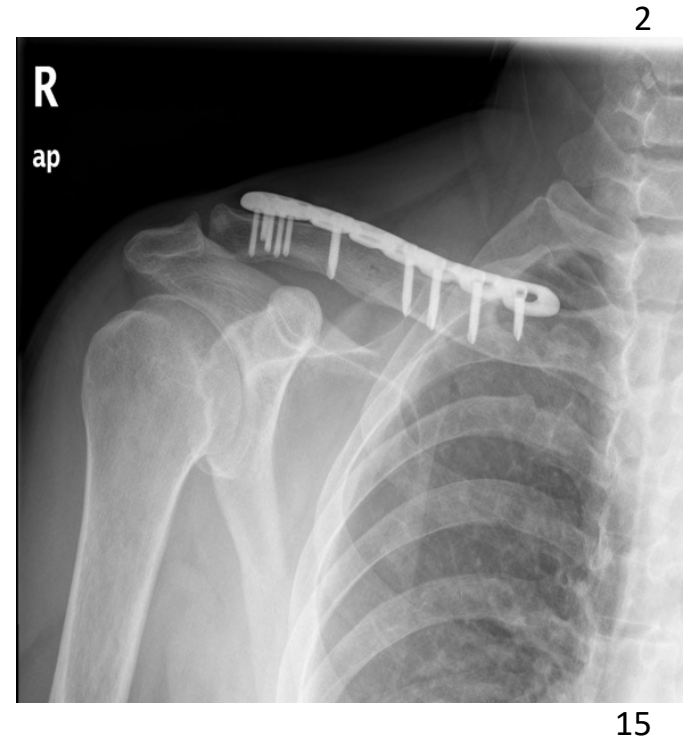

16

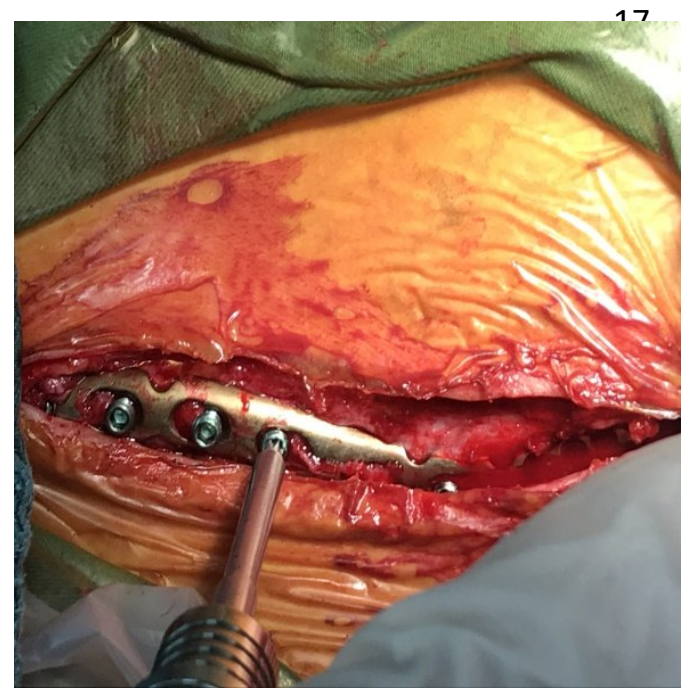

Fig.4

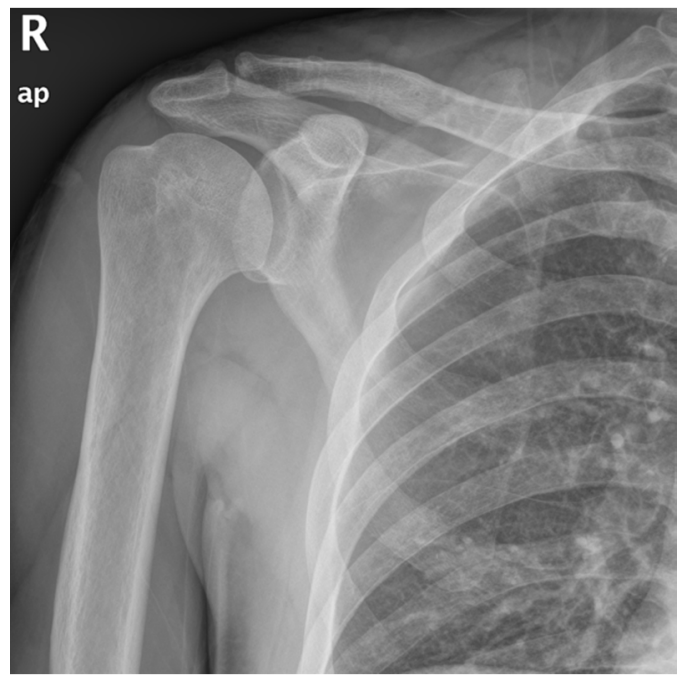

Fig.3

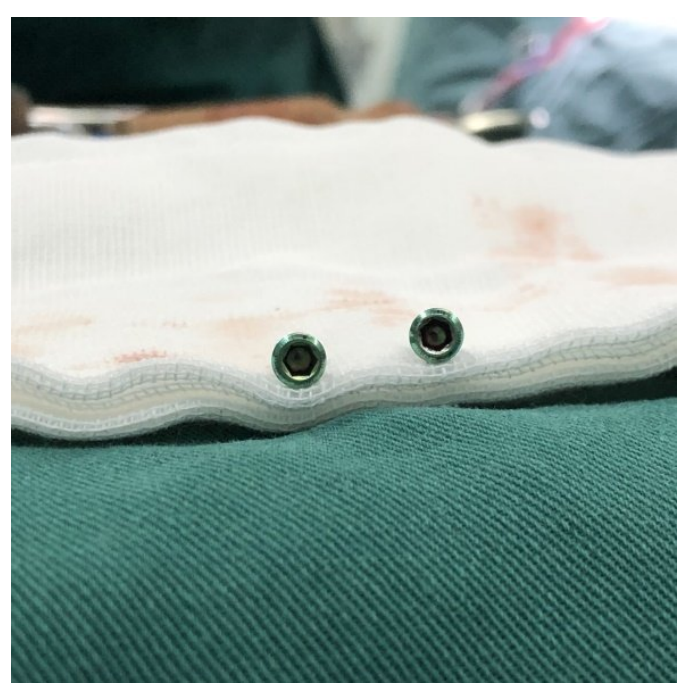

Fig.5

Fig.2-5: The typical case: A 55-year-old male with postoperative bone union of right clavicle fracture.Fig.2-3: Positive position X-ray film of right clavicle before or after operation. Fig4-5: the slide wire screw was removed by nail groove reconstruction method (1)

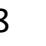
(1) 

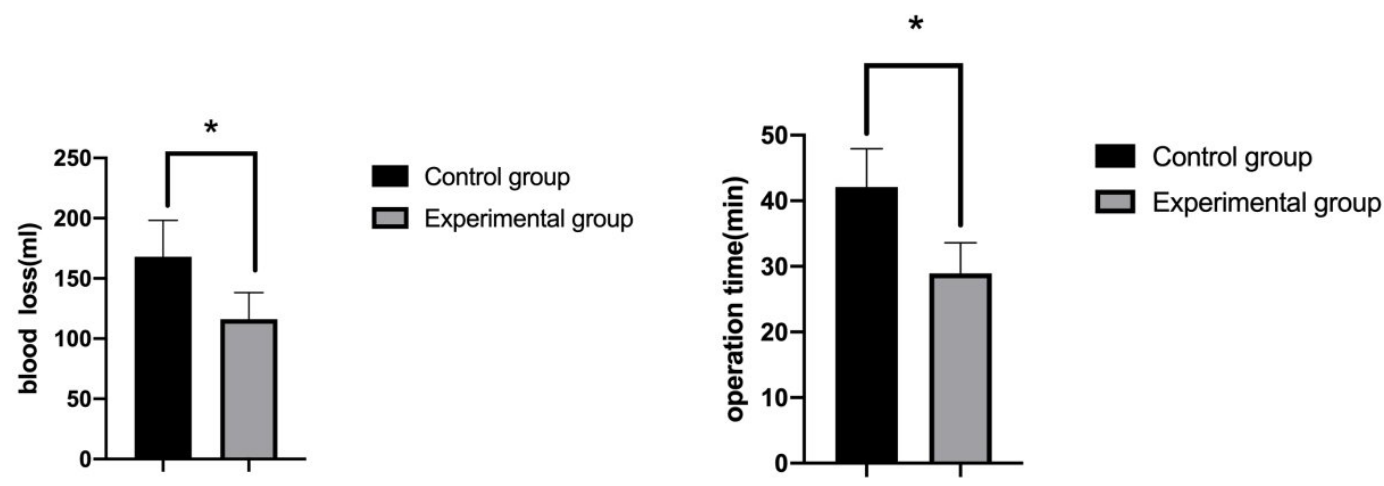

2

(b)

4

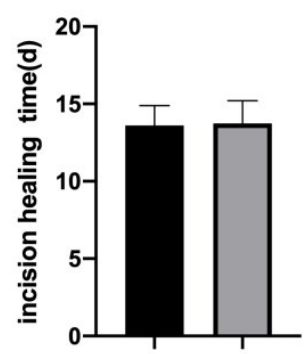

Control group
Experimental group

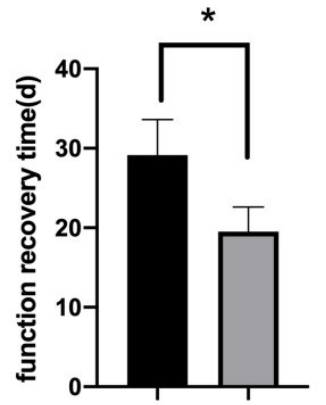

Control group

Experimental group

5

(c)

(d)

7

Fig.6 Comparison of perioperative indicators between the two groups

(a) The quantity of blood loss to remove a slide wire screw of two groups

(b) The time it takes to remove a slide wire screw of two groups

(c) The number of days for incision to get grade A healing after operation of two groups

(d) The number of days for the limb to recovery preoperative function of two groups

(Note: * Compared with the control group, $\mathrm{P}<0.05$ ) 